\section{(6) OPEN ACCESS}

\title{
Effectiveness of a brief community outreach tobacco cessation intervention in India: a cluster-randomised controlled trial (the BABEX Trial)
}

\author{
Bidyut K Sarkar, ${ }^{1,2}$ Robert West, ${ }^{2}$ Monika Arora, ${ }^{1}$ Jasjit S Ahluwalia, ${ }^{3}$ \\ K Srinath Reddy, ${ }^{1}$ Lion Shahab ${ }^{2}$
}

${ }^{1}$ Public Health Foundation of India, New Delhi, India

${ }^{2}$ Department of Epidemiology and Public Health, Cancer

Research UK Health Behaviour Research Centre, University College London, London, UK ${ }^{3}$ School of Public Health, Rutgers University, New Brunswick, USA

Correspondence to Dr Bidyut K Sarkar, Public Health Foundation of India, New Delhi 110070, India; bidyutk.sarkar@gmail.com

Received 5 April 2016 Revised 2 August 2016 Accepted 7 September 2016 Published Online First 5 October 2016

\section{SLlinked}

http://dx.doi.org/10.1136/ thoraxjnl-2016-209435 CrossMark

To cite: Sarkar BK, West R, Arora $\mathrm{M}$, et al. Thorax 2017;72:167-173.

\section{ABSTRACT}

Background Tobacco use kills half a million people every month, most in low-middle income countries (LMICS). There is an urgent need to identify potentially low-cost, scalable tobacco cessation interventions for these countries.

Objective To evaluate a brief community outreach intervention delivered by health workers to promote tobacco cessation in India.

Design Cluster-randomised controlled trial.

Setting 32 low-income administrative blocks in Delhi, half government authorised ('resettlement colony') and half unauthorised ('J.J. cluster') communities.

Participants 1213 adult tobacco users.

Interventions Administrative blocks were computer randomised in a 1:1 ratio, to the intervention (16 clusters; $\mathrm{n}=611$ ) or control treatment ( 16 clusters; $n=602$ ), delivered and assessed at individual level between 07/2012 and 11/2013. The intervention was single session quit advice (15 min) plus a single training session in yogic breathing exercises; the control condition comprised very brief quit advice (1 $\mathrm{min}$ ) alone. Both were delivered via outreach, with contact made though household visits.

Measurements The primary outcome was 6-month sustained abstinence from all tobacco, assessed 7 months post intervention delivery, biochemically verified with salivary cotinine.

Results The smoking cessation rate was higher in the intervention group $(2.6 \%(16 / 611))$ than in the control group $(0.5 \%(3 / 602))$ (relative risk $=5.32,95 \% \mathrm{Cl} 1.43$ to $19.74, p=0.013)$. There was no interaction with type of tobacco use (smoked vs smokeless). Results did not change materially in adjusted analyses, controlling for participant characteristics.

Conclusions A single session community outreach intervention can increase tobacco cessation in LMIC. The effect size, while small, could impact public health if scaled up with high coverage.

Trial registration number ISRCTCN23362894.

\section{INTRODUCTION}

Tobacco use causes six million premature deaths each year, most in low-income and middle-income countries (LMICs), ${ }^{1}$ and one million in India alone. ${ }^{2}$ Even small increases in long-term tobacco cessation rates can have an important public health impact. ${ }^{3}$ Combatting the tobacco epidemic requires a comprehensive, global strategy including taxation,

\section{Key messages}

What is the key question?

- Does a potentially low-cost and scalable community-outreach intervention, single session advice (15 $\mathrm{min}$ ) to stop tobacco use combined with training in yogic breathing exercise, increase abstinence rates from tobacco use in the context of a low-middle income country (LMIC) compared with very brief quit advice (1 $\mathrm{min}$ ) alone?

\section{What is the bottom line?}

- A simple, cheap and potentially scalable community-outreach intervention increases smoked and smokeless tobacco cessation in India, producing an effect size similar to that of brief physician advice in high-income countries.

\section{Why read on?}

- Given the number of tobacco users and limited resources in LMIC, there is a need to develop cheap and scalable interventions to combat tobacco use and, to our knowledge, this is the first trial to establish the effectiveness of a single session community outreach intervention to stop tobacco use in low-income communities in any LMIC worldwide.

smoke-free policies, and dedicated healthcare services to encourage and support cessation. ${ }^{4}$ However, implementation in LMICs has been limited, hampered by weak regulatory and enforcement infrastructure. ${ }^{5}$ Cheap, scalable tobacco cessation interventions that build on existing infrastructure could strengthen tobacco control in the Indian context. ${ }^{6}$

Brief opportunistic advice from a health worker has been found to be a highly cost-effective intervention to promote smoking cessation in highincome countries (HICs), ${ }^{7}$ increasing 12-month quit rates by one to three percentage points ${ }^{8}$ and offering assistance is an important part of the process. ${ }^{9}$ However, there is limited evidence on the effectiveness of quit advice in LMICs, from nonphysicians, or for smokeless tobacco use. These represent important gaps in the evidence base. 
This paper attempts to fill these gaps, evaluating an intervention of single session smoked and smokeless tobacco cessation advice plus a single session of yogic breathing exercises, delivered by healthcare professionals via community outreach in India.

Tobacco use, a major cause of health inequalities, is more prevalent and quit success rates are lower in deprived groups. ${ }^{10}$ Even within poorer countries, there is a gradient in tobacco use. ${ }^{11}$ While there is a need to improve support across all societal strata in LMICs, arguably, it is particularly important to focus on low-income communities within those countries by outreach to involve tobacco users who would not otherwise come into contact with health services. ${ }^{6}$

Given limited resources, and a lack of affordable pharmacotherapy with proven effectiveness for smokeless tobacco users, providing assistance presents a challenge in LMICs such as India with more smokeless tobacco users than smokers. ${ }^{12} \mathrm{~A}$ promising and culturally appropriate option in this context is the use of yogic breathing exercises, known as 'Pranayama'. These are easy to learn and practice, and there is preliminary evidence they can reduce cigarette cravings ${ }^{13}{ }^{14}$ and promote quitting. ${ }^{15}$ We therefore developed a brief community outreach intervention that included training on simple breathing exercises to control cravings to aid cessation.

Ideally, an evaluation of a multi-component intervention would involve multiple experimental conditions allowing determination of active components. Unfortunately, with limited resources the main priority is to find an intervention that works. Understanding how it can be optimised can be done once this 'base camp' has been established. Therefore we opted for a pragmatic, effectiveness trial of an intervention compared with the closest to 'usual care' that was ethically acceptable, undertaken in conditions mimicking what is feasible in routine practice. Cluster rather than individual randomisation was chosen to minimise risk of contamination given the close-knit nature of these communities. Both treatment delivery and assessment were at an individual level. Following guidelines on complex intervention development and evaluation, ${ }^{16}$ this study built on the extensive work on brief advice from HIC, and proof of concept studies on breathing exercises as well as field studies in this particular setting. ${ }^{17}$ Specifically, the present study sought to answer the following research question: how effective is single session quit advice $(15 \mathrm{~min})$ with instruction on simple breathing exercises compared with very brief advice (VBA) alone (1 min) in promoting tobacco cessation when delivered by outreach workers in low-income communities in India?

\section{METHODS}

\section{Study design}

This pragmatic, cluster-randomised controlled trial set in lowincome communities (urban slums) compared two treatments: the intervention arm included a single face-to-face session of quit advice plus a single training session on yogic breathing exercises (BA-YBE); the control arm comprised VBA alone. Assessments were performed at baseline (immediately before treatment delivery), 4 weeks and 7 months after delivery of the treatments. The study was approved by the UCL $(3051 / 002)$ and Public Health Foundation of India (TRC-IEC-122/11) ethics committees.

\section{Participants}

Eligible participants were any current, daily, adult tobacco user aged 23 years or above, living in selected low-income communities, who provided consent to participate. Recruitment into the study started in July 2012 and the trial concluded in November
2013. The proposed study area contained approximately 36000 adults living in 32 randomly selected administrative blocks of large communities spread over a wide geographical area in Delhi, India. These low-income communities had been selected for a previous tobacco study conducted among youths aged 10 19 years in $2009,{ }^{18}$ therefore only adults aged 23 or above were eligible to avoid contamination with the previous study.

\section{Intervention}

Participants in the intervention condition received a single session of tobacco quit advice lasting an average of $15 \mathrm{~min}$, and short training in two yogic breathing exercises. The quit advice contained behaviour change techniques which have been shown to improve quit rates, ${ }^{19}$ including coping training, medication advice, social support and relapse prevention. Two breathing exercises which are easy to learn and practise, 'Kapalbhati' (normal deep inhalation-forceful exhalation) and 'Anulom vilom' (alternate nostril breathing), were chosen as they are culturally appropriate and have been shown to control cravings. ${ }^{14}$ A written standard operating procedure was followed by the research team, including a script for quit advice and a standard video for training on yogic breathing exercises to increase fidelity. Further details are available in the published protocol. ${ }^{20}$

\section{Control}

Whilst a control condition with no advice on tobacco use ('usual care') would be preferable to assess the full intervention effect, this was considered unethical. Therefore a single control session involving very brief quit advice ${ }^{21}$ was used. This provided verbal information about the harmful effects of tobacco use and advice to stop tobacco use and lasted on average $1 \mathrm{~min}$.

\section{Procedure, randomisation and masking}

A census survey of adults in urban low-income communities of Delhi was conducted to establish an appropriate sampling frame for this study. ${ }^{17}$ As low-income blocks in the study area are stratified into two community types (government authorised 'resettlement colonies' and unauthorised settlements called 'Jhuggi-Jhopri' or 'JJ clusters'), an equal number of both were identified for inclusion. The clusters were identified and a list of all eligible tobacco users finalised within each cluster prior to randomisation of clusters to the intervention or control arm. The random sequence was computer generated and blocked to ensure equal numbers of each community type in the intervention and control conditions. Based on the random sequence, 16 clusters (8 from each community type) were allocated to the treatment (BA-YBE) and control (VBA) arm, respectively. While participants were blind to allocation, being a cluster-randomised trial, concealment of allocation to the research team was not feasible.

The baseline interview was conducted at the doorstep of potential participants and those identified as tobacco users were invited to participate. Each cluster was divided into three to four geographical parts (streets/lanes/outer/inner) comprising an equal number of households from which an equal number of participants were approached. Those willing to provide consent were checked for eligibility and formal written consent was obtained. In the case of multiple eligible participants in the same household, the first consenting tobacco user identified in the sampling frame was recruited.

The treatment delivery team consisted of two members, a medical graduate researcher (physician) and a field investigator (community health worker) trained and initially supervised by the medical graduate researcher. The intervention was delivered 
by either of them. The field investigators were given 1 day $f$ classroom training for intervention delivery using standard tools like a script for quit advice and use of a standard video for training in yogic breathing exercises. All content was delivered face to face in non-technical, local language (Hindi) in both groups. Follow-up visits comprised face-to-face interviews at the residence of participants, 4 weeks and 7 months after the baseline visit. Due to cluster randomization, blinding was not feasible but follow-up was carried out by a different team of field investigators.

\section{Measures}

All measures, including outcome measures, were pre-specified and defined in the ISRCTN Registry and published protocol. ${ }^{20}$ The primary outcome measure, 6-month sustained abstinence, was defined as per Russell Standard (RS) criteria, ${ }^{22}$ allowing a maximum of five instances of tobacco use (in whichever form) in the 6 months preceding the follow-up conducted 7 months after treatment delivery, and was biochemically validated. As the sample included smokeless and smoked tobacco users, salivary cotinine was used to detect tobacco exposure, assessed by ELISA with a cut-off of $20 \mathrm{ng} / \mathrm{mL}$, equivalent to the $13 \mathrm{ng} / \mathrm{mL}$ used for the gas chromatography mass spectrometry method. ${ }^{23}$ Self-reported abstainers above the cut-off were considered to be continuing tobacco users as were participants lost to follow-up, in accordance with the intention-to-treat principle and RS criteria. ${ }^{22}$ Nicotine replacement therapy (NRT) and other medication use was assessed at follow-up to exclude false-positive cotinine results.

The secondary outcome measure was 7-day biochemically validated point prevalence abstinence assessed 7 months after treatment delivery. At the 4-week follow-up, all participants were asked whether they had used tobacco in the past 7 days (7-day point prevalence), and intervention group participants were also asked how often they had used the yogic breathing exercises and how useful they had found them on a seven-point scale, ranging from 'not at all' to 'very helpful'.

At baseline, standard socio-demographic and smoking characteristics were assessed. In addition to age, sex and marital status, participants were asked about employment status (employed vs other), highest educational attainment (at least primary education vs not), caste (lower caste vs other) and household income $(\leq 5000$ vs $>5000$ rupees/month). Participants were asked when they started tobacco use, dependence (assessed with the heaviness of smoking index,${ }^{24}$ modified for smokeless tobacco users), quit attempts in the past year, length of the attempt and whether support was used as well as how confident they felt about stopping smoking on a sevenpoint scale ranging from 'not at all confident' to 'very confident'. Lastly, adverse and serious adverse events were monitored and recorded by the medical graduate researcher throughout the study.

\section{Sample size}

The initial power calculation based on 32 clusters indicated that 31 participants per cluster would be needed for $90 \%$ power to detect a $5 \%$ difference in quit rates between the treatment and control group in two-tailed analysis $(7 \%$ vs $2 \%){ }^{20}$ The effect estimate was based on early, similar work ${ }^{25}$ and assumed an intra-cluster correlation co-efficient (ICC) of 0.01 , typical in this kind of study, and a design effect of 1.24 to account for cluster randomisation. However, as low-intensity interventions can have substantial attrition rates (in trials using evidence-based methods to increase response rates, dropout rates of $20-25 \%$ at 6-month follow-up are common) ${ }^{26}$ which reduces the power to detect effects in an intention-to-treat design ${ }^{27}$ and due to the uncertainty around the ICC estimate, we over-recruited by $20 \%$ per cluster (37.2 participants, rounded up to 38 participants), resulting in a final target sample size of 1216 ( $38 \times 32$ clusters). This sample size also provided $90 \%$ power to detect a larger difference $(8 \%)$ in subgroup analyses.

\section{Analysis}

Primary and secondary smoking cessation outcomes and baseline group differences were analysed with a mixed-effects logbinomial or linear regression with appropriate link functions for categorical ( $\log / \operatorname{logit}-b i n o m i a l)$ and continuous (identity Gaussian) outcome variables using the 'Ime4' package in $\mathrm{R}$ (V.3.2.0) (Bates DM. Ime4: Mixed-effects modelling with R: Springer, 2010) to account for clustered observations, which is preferable to the protocol-specified complex samples analysis. ${ }^{28}$ All other analyses were conducted in STATA (V.13.1). Results from the conditional/cluster-specific models were confirmed with marginal/population-averaged models using generalised estimating equations ('xtgee' command) with an exchangeable correlation matrix to account for clustering, ${ }^{29}$ and ICC was determined using the analysis of variance method. ${ }^{30}$

In planned subgroup analyses, intervention by participant (smoked vs smokeless tobacco and dual product users) and intervention by provider (trained health professional vs trained field health workers) interaction terms were included in models to assess homogeneity of the effect across these subgroups. In sensitivity analysis, we analysed only those who actually received the treatment and effect estimates were adjusted for covariates to account for chance imbalances in baseline characteristics between intervention and control conditions. Significance levels for multiple comparisons were adjusted with the Benjamini-Hochberg procedure. ${ }^{31}$ The study was registered with the International Standard Randomised Controlled Trial Number Registry (number ISRCTCN23362894).

\section{RESULTS}

As shown in figure 1, 85.9\% (1213/1412) of eligible tobacco users approached between January 2012 and November 2013 agreed to take part in the study; the most common reason for not taking part was lack of interest. The follow-up rate at final endpoint was high at 95.3\% (1158/1213) and did not differ between arms. There were no differences in baseline characteristics between those who were and were not followed up.

The sample of tobacco users recruited was largely male, middle aged, married, in employment, with at least a primary education, most were relatively deprived as measured by household income and nearly half were from the lower caste (table 1). There were slightly more tobacco smokers than smokeless tobacco users; a substantial proportion were dual users. Most had been using tobacco products for 20 years and were moderately dependent. A fifth had made a quit attempt in the last year, and past quit attempts had lasted up to 2 months, but very few had previously used any quit support. Participants were generally confident in their ability to quit. There were no chance imbalances between groups with the exception of caste: significantly more participants in the intervention than control group were lower caste (table 1).

Regarding the primary outcome, 2.6\% (16/611) of participants were continuously abstinent at 6 months as per RS criteria, validated by saliva cotinine. Intention-to-treat analysis showed that participants in the intervention group were about five times more likely to be abstinent at 6 months than those in 


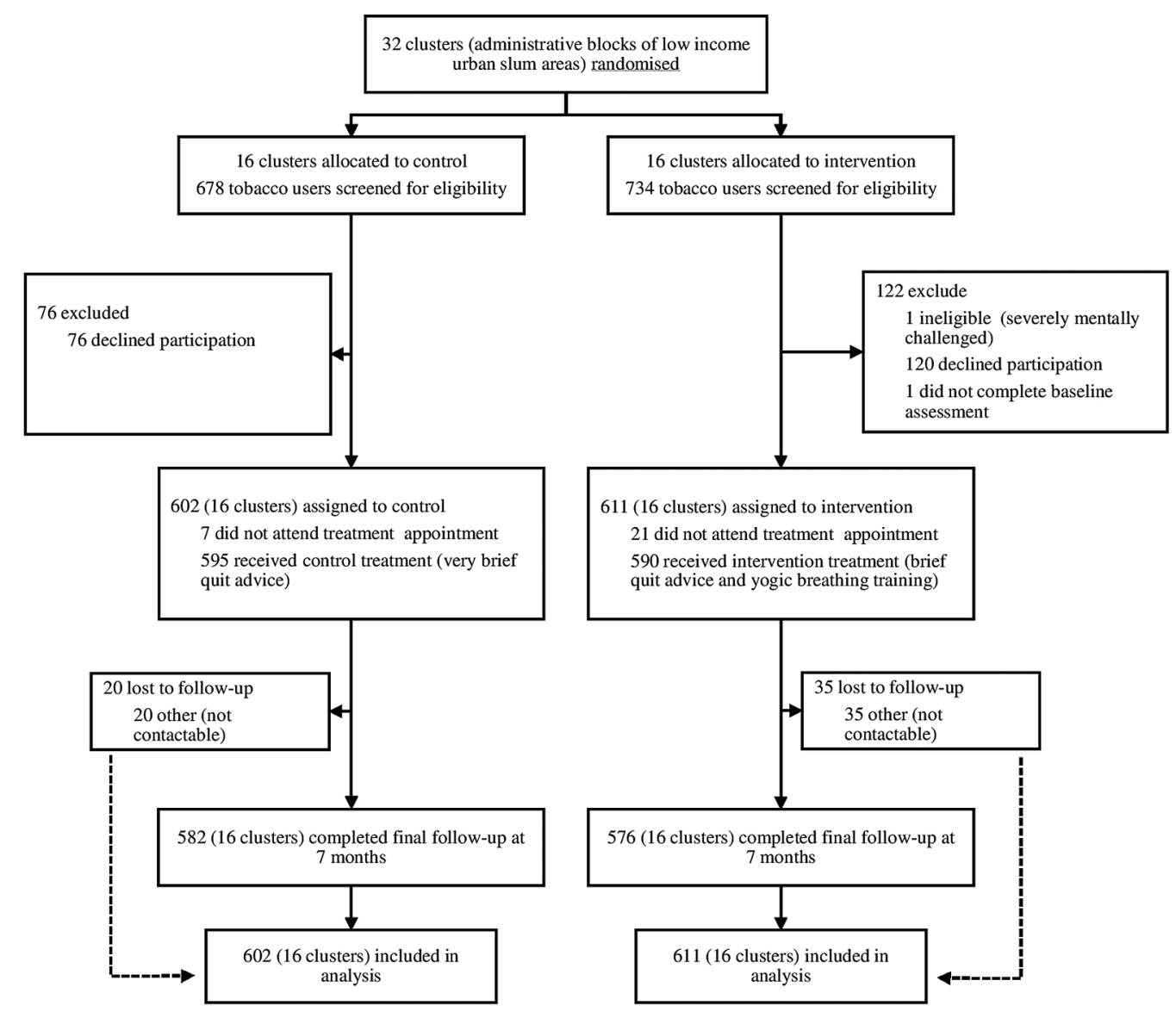

Figure 1 Numbers of participants and clusters enrolled in the study and included in the primary analysis.

the control group; absolute abstinence rates were increased by $2 \%$ (table 2), yielding a number needed to treat of 48 to produce an additional quitter. This difference did not materially alter and remained significant when controlling for all other covariates.

Validated 7-day point prevalence at 6 months in the total sample was marginally higher at $2.0 \%$ (24/1213). Participants in the intervention group were nearly four times more likely to achieve 7-day abstinence at 6 months than those in the intervention group and as before results did not materially change when controlling for other covariates (table 2).

Overall self-reported 7 -day point prevalence at 4 weeks was $9.3 \%(113 / 1213)$. As with the primary and secondary outcomes, abstinence rates were significantly higher in the intervention group than the control group, more than doubling (RR 2.03; $95 \%$ CI 1.30 to $3.19 ; \mathrm{p}=0.002)$ to $12.4 \%(76 / 611)$ from $6.1 \%$ (37/602). At 4-week follow-up, 61.1\% (373/611) in the intervention group had continued to use the breathing exercises, a fifth $(19.0 \%(116 / 611))$ on most days. They rated the breathing exercises as helpful (mean $=5.3, \mathrm{SD}=1.8$ ). No adverse or serious adverse events were reported.

In pre-specified per protocol analyses of the primary outcome, we analysed data from participants who had actually received treatment, given that significantly more participants in the intervention group than the control group $(3.4 \%(21 / 611)$ vs $1.2 \%(7 / 602) ; \mathrm{p}=0.03)$ missed their treatment. This did not alter the effect of the intervention (RR 5.48; 95\% CI 1.46 to $20.51 ; \mathrm{p}=0.01)$. We also included potential effect modifiers, the type of tobacco used (smoked vs smokeless, excluding dual users), poly-use (dual vs single use) and intervention provider (trained health professional vs trained field health workers), as interaction terms in the main model to assess whether effects differed between these groups. There was no evidence that type of product used $\left(p_{\text {interaction }}=0.32\right)$, poly-use $\left(p_{\text {interaction }}=0.85\right)$ or the intervention provider $\left(p_{\text {interaction }}=0.90\right)$ affected the outcome.

\section{DISCUSSION}

In a trial of tobacco users from low-income communities in India, 6-month sustained biochemically verified abstinence rates were increased fivefold by a low-cost intervention combining single session quit advice with yogic breathing exercises when compared with very brief quit advice alone. The increase of $2 \%$ in absolute quit rates is close to that obtained by other lowintensity interventions observed in HIC. ${ }^{8} 33$ These results also compare favourably with previous evaluations of more intensive interventions in LMIC which roughly double shortterm abstinence rates from tobacco. ${ }^{5}$ While the absolute quit rates were lower than expected, this may not only reflect the simplicity of the intervention which did not provide any pharmacological support but also the broader challenge of making behaviour change interventions relevant in the context of extreme deprivation, as has been observed elsewhere. ${ }^{35}$ However, since it is non-physician based, it is potentially scalable in settings like India with an inadequate healthcare delivery system especially for populations with limited access to physicians or medications. It is noteworthy that the average family income of participants was $£ 50$ per month or less than $£ 2$ per day, which makes even NRT unaffordable unless funded by the government. At follow-up, use of NRT and other medications 
Table 1 Baseline characteristics of participants

\begin{tabular}{|c|c|c|c|}
\hline Characteristics & Total $(\mathrm{N}=1213)$ & $\begin{array}{l}\text { Intervention (BA-YBA)* } \\
(\mathrm{N}=611)\end{array}$ & $\begin{array}{l}\text { Control (VBA) } t \\
(\mathrm{~N}=602)\end{array}$ \\
\hline \multicolumn{4}{|l|}{ Sociodemographic } \\
\hline$\%(\mathrm{~N})$ Male & 79.7 (966) & $77.4(472)$ & $82.1(494)$ \\
\hline Mean (SD) age in years & $46.3(13.6)$ & $45.2(12.8)$ & $47.4(14.2)$ \\
\hline$\%(\mathrm{~N})$ Married/cohabiting $\ddagger$ & $86.4(1044)$ & $85.2(517)$ & $87.5(527)$ \\
\hline$\%(\mathrm{~N})$ In employment§ & $70.7(856)$ & $71.7(438)$ & $69.7(418)$ \\
\hline$\%(\mathrm{~N})$ No primary education 9 & $35.3(426)$ & $33.3(202)$ & $37.3(224)$ \\
\hline$\%$ (N) Household income $\geq 5000$ INR/month** & $34.1(403)$ & $35.7(212)$ & $32.5(191)$ \\
\hline$\%(\mathrm{~N})$ Lower castet† & $43.8(512)$ & $50.7(290)$ & $37.2(222)$ \\
\hline \multicolumn{4}{|l|}{ Tobacco use } \\
\hline$\%(\mathrm{~N})$ Smokers & $63.8(774)$ & $65.0(397)$ & $62.6(377)$ \\
\hline$\%(\mathrm{~N})$ Smokeless users & $58.5(710)$ & $58.8(359)$ & $58.3(351)$ \\
\hline$\%(\mathrm{~N})$ Dual users & $22.3(271)$ & $23.7(145)$ & $20.9(126)$ \\
\hline Mean (SD) age started use $\ddagger$ & $22.4(9.6)$ & $22.2(10.0)$ & $22.6(9.3)$ \\
\hline$\%(\mathrm{~N})$ Made quit attempt in the previous year§§ & $20.0(229)$ & $22.3(126)$ & $17.8(103)$ \\
\hline Mean (SD) length of longest quit attempt (in weeks) & $7.6(30.0)$ & $7.5(33.2)$ & $7.8(26.5)$ \\
\hline$\%(\mathrm{~N})$ Previously used support in a quit attempt ${ }^{* * *}$ & $3.8(46)$ & $3.8(23)$ & $3.8(23)$ \\
\hline Mean (SD) heaviness of smoking index score $(0-6)+\dagger \dagger$ & $3.0(1.7)$ & $3.2(1.6)$ & $2.8(1.7)$ \\
\hline 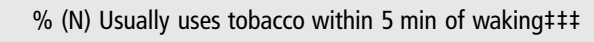 & $38.0(458)$ & $40.0(243)$ & $36.0(215)$ \\
\hline Mean (SD) confidence in stopping score $(1-7) \S \S \S$ & $4.4(1.9)$ & $4.5(1.9)$ & $4.2(2.0)$ \\
\hline 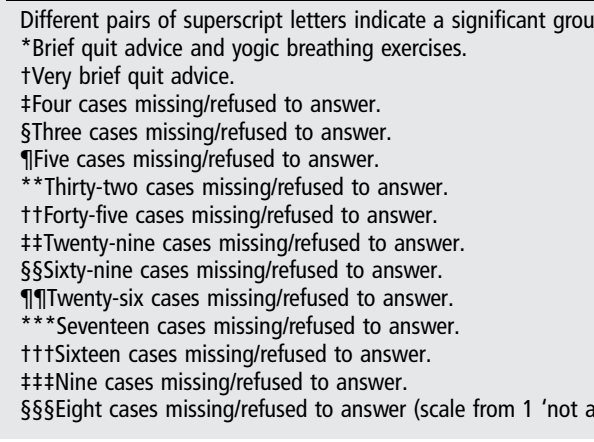 & 05) after adjustment & rate and accounting for clus & \\
\hline
\end{tabular}

Table 2 Effect of intervention on biochemically verified smoking cessation*

\begin{tabular}{|c|c|c|c|c|c|}
\hline & $\begin{array}{l}\text { Intervention } \\
\text { (BA-YBE) }\end{array}$ & $\begin{array}{l}\text { Control } \\
\text { (VBA) }\end{array}$ & & & \\
\hline & $\%$ (number) & & $\begin{array}{l}\text { Percentage point } \\
\text { difference } \\
(95 \% \mathrm{Cl})\end{array}$ & $\begin{array}{l}\text { Intracluster correlation } \\
\text { coefficient } \\
(95 \% \mathrm{Cl})\end{array}$ & $\begin{array}{l}\text { Model 1: Relative risk† } \\
\text { Model 2: Adj. relative riskł } \\
\text { Model 3: Adj. relative risk§ } \\
(95 \% \mathrm{Cl})\end{array}$ \\
\hline $\begin{array}{l}\text { Primary outcome: abstinence } \\
\text { for } 6 \text { months } 1\end{array}$ & $2.6(16 / 611)$ & $0.5(3 / 602)$ & $2.12(0.74$ to 3.51$)$ & $0.014(0.000$ to 0.033$)$ & $\begin{array}{l}\text { Model 1: } 5.32(1.43 \text { to } 19.74) \text { ** } \\
\text { Model 2: } 5.10(1.46 \text { to } 17.84) \text { ** } \\
\text { Model 3: } 4.54(1.21 \text { to } 17.01) \text { t† }\end{array}$ \\
\hline $\begin{array}{l}\text { Secondary outcome: point } \\
\text { prevalence at } 6 \text { months } \ddagger\end{array}$ & $3.1(19 / 611)$ & $0.8(5 / 602)$ & $2.28(0.72$ to 3.83$)$ & $0.014(0.000$ to 0.034$)$ & $\begin{array}{l}\text { Model 1: } 3.77(1.31 \text { to } 10.81) \text { ** } \\
\text { Model 2: } 3.71(1.25 \text { to } 11.02) \S \S \\
\text { Model 3: } 2.87(0.92 \text { to } 8.93) \text { ๆी }\end{array}$ \\
\hline \multicolumn{6}{|c|}{ 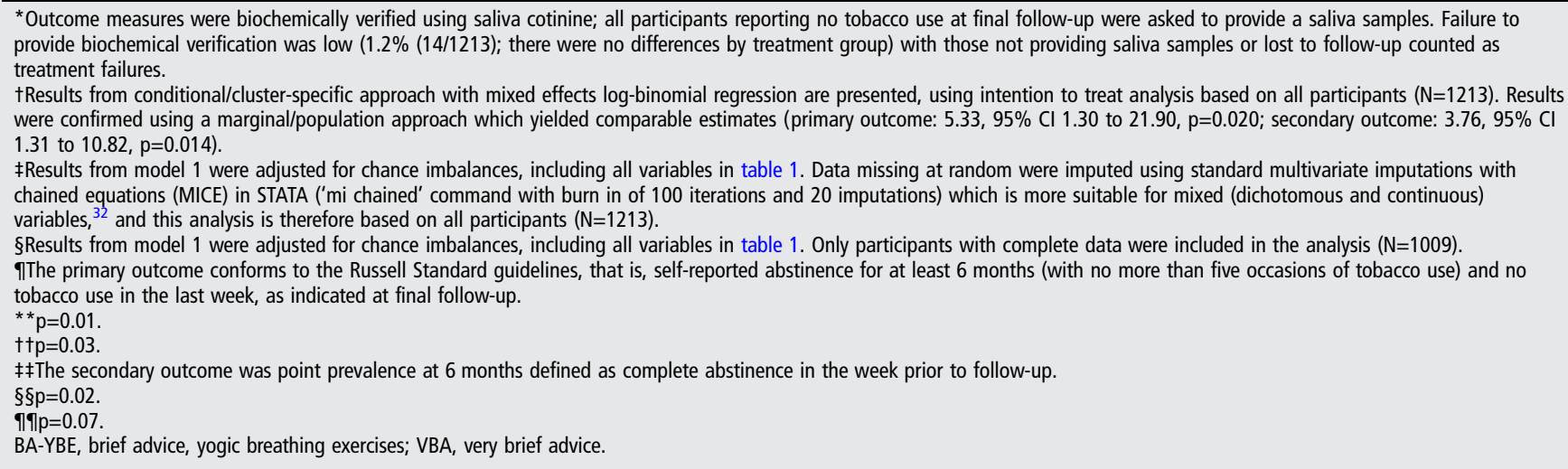 } \\
\hline
\end{tabular}


for quitting was assessed but not reported by any participants, likely due to prohibitive costs. ${ }^{36}$

To our knowledge, this is the first trial using a rigorous design, including longer-term, biochemically validated outcomes to establish the effectiveness of brief, pro-active quit advice in low-income communities in any LMIC. Given that community health workers, who earn around $£ 100-150$ per month, were given just 1 day training to deliver the intervention, that the intervention was equally effective when provided either by a community health worker or by a physician researcher and that total study material costs were below $£ 200$, this intervention is likely to also be highly cost effective at a cost of less than $£ 10$ per treated tobacco user. ${ }^{37}$

This study benefited from assessing an evidence-based and theory-based intervention using standardised methodology, including validated longer-term outcomes, extensive prior field work and low attrition rates. A number of factors may have contributed to the effectiveness and reach of the intervention. First, yogic breathing exercises in addition to reducing cravings, ${ }^{14}$ possibly via actions in the insula in the cerebral cortex, ${ }^{38}$ may also appeal due to being a novel, culturally acceptable cessation aid which provides an element of support without the need for referral or expensive medications. Second, the proactive, convenient delivery of the intervention near participants' homes may have engaged more tobacco users. Third, the treatment being free of charge may have drawn in tobacco users, irrespective of their readiness to quit. Lastly, the advice, being centred around evidence-based content, ${ }^{19}$ was tailored to the specific cultural context, targeting users of both smoked and smokeless tobacco, and-as evidenced by very limited smoking-related knowledge at baseline (not reported here)-provided extensive details hitherto unknown to participants.

There were some limitations. Since the intervention comprised both quit advice and training in yogic breathing exercises, the effect of specific intervention components cannot be dissociated. However, the priority at this stage was to identify a potentially cost-effective, scalable brief community outreach intervention that included effective, research-based components. Future factorial studies should further isolate the impact of different intervention components on outcome. The number of events in this trial was small. Although this is not uncommon in low-intensity interventions, replication of findings is warranted. Lastly, whilst every effort was made to select a random sample, findings are necessarily limited to this sample of deprived tobacco users in Delhi. However, given that outcomes were comparable to those from a study evaluating a similarly brief, two-session tobacco use intervention in rural India, ${ }^{39}$ the results may be generalised to other geographic locations and LMICs. Nonetheless, further work should confirm whether this type of opportunistic, brief quit advice and yogic breathing exercises is equally effective in other relevant settings, such as schools or work places, to maximise its potential impact.

In conclusion, we have shown a substantial effect of a potentially inexpensive, scalable, non-physician-dependent, culturally sensitive intervention to aid cessation of tobacco use in LMICs. Although the number needed to treat to gain an additional quitter was high at 48, the low intervention cost and ease of implementation with limited additional resources (see protocol for details) ${ }^{20}$ means that it may offer a credible alternative to more effective but also more expensive and intensive treatments, such as pharmacotherapy, with very low coverage, to tackle the enormous tobacco problem facing disadvantaged users in some of the poorest countries of the world. Despite the small effect observed, even minimal changes in tobacco use can have clinical significance. ${ }^{3}$ Indeed, if this intervention were to be rolled out across India, it would likely result in several million fewer tobacco users per year. While further research is needed to confirm our findings in different settings, the current trial is consistent with results from HIC and underlines the great potential of low-intensity interventions to improve public health in the specific context of LMICs.

Acknowledgements The authors would like to thank the field investigators and staff of NGO HRIDAY for their hard work and excellent support for the field work and data collection, as well as all the tobacco users who consented to participate, without whom this study would not have been possible.

Contributors BKS, LS and RW conceived the original idea for this RCT. BKS obtained funding. BKS, LS and RW wrote the study protocol for the RCT reported in this manuscript. BKS managed the day-to-day running of the trial, including all participant follow-up. BKS, RW and LS undertook the data analyses and wrote the initial draft with further input from MA, JSA and KSR. BKS is guarantor for this article. All authors read, reviewed and approved the final version. All researchers listed as authors are independent from the funders and all final decisions about the research were taken without constraint by the investigators. BKS, RW and LS had full access to all the data in the study and BKS had final responsibility for the decision to submit for publication.

Funding This study was supported by a Wellcome Trust, UK Capacity Strengthening Strategic award to Public Health Foundation of India (PHFI) and a consortium of UK Universities (Award no 6936), with additional funding from Cancer Research UK (C1417/A14135) and UK's National Centre for Smoking Cessation and Training. The funders of the study had no role in study design, data collection, data analysis, data interpretation or writing of the report. The corresponding author had full access to all the data in the study and had final responsibility for the decision to submit for publication.

Competing interests $L S$ reports personal fees from pharmaceutical companies that make smoking cessation products and grants from Pfizer, outside the submitted work. RW reports grants and personal fees from companies that develop and manufacture smoking cessation medications, outside the submitted work; in addition, he has a dormant patent 'Nicotine cannon' (novel nicotine delivery device) issued.

Ethics approval The study was approved by the UCL (3051/002) and Public Health Foundation of India (TRC-IEC-122/11) ethics committees.

Provenance and peer review Not commissioned; externally peer reviewed.

Open Access This is an Open Access article distributed in accordance with the terms of the Creative Commons Attribution (CC BY 4.0) license, which permits others to distribute, remix, adapt and build upon this work, for commercial use, provided the original work is properly cited. See: http://creativecommons.org/licenses/ by/4.0/

\section{REFERENCES}

1 World Health Organization. WHO global report: mortality attributable to tobacco. Geneva: WHO, 2012.

2 Jha $\mathrm{P}$, Jacob B, Gajalakshmi V, et al. A nationally representative case-control study of smoking and death in India. N Engl J Med 2008;358:1137-47.

3 West R. The clinical significance of "small" effects of smoking cessation treatments. Addiction 2007;102:506-9.

4 World Health Organization. WHO report on the global tobacco epidemic, 2008: the MPOWER package. Geneva, Switzerland: WHO, 2009.

5 McKay AJ, Patel RK, Majeed A. Strategies for tobacco control in India: a systematic review. PLOS ONE 2015;10:e0122610

6 Sarkar BK, Reddy KS. Priorities for tobacco control research in India. Addiction 2012;107:2066-8.

7 West R, Raw M, McNeill A, et al. Health-care interventions to promote and assist tobacco cessation: a review of efficacy, effectiveness and affordability for use in national guideline development. Addiction 2015;110:1388-403.

8 Stead LF, Buitrago D, Preciado N, et al. Physician advice for smoking cessation. Cochrane Database Syst Rev 2013;5:CD000165.

9 Aveyard P, Begh R, Parsons A, et al. Brief opportunistic smoking cessation interventions: a systematic review and meta-analysis to compare advice to quit and offer of assistance. Addiction 2012;107:1066-73.

10 Vangeli E, Stapleton J, Smit ES, et al. Predictors of attempts to stop smoking and their success in adult general population samples: a systematic review. Addiction 2011; 106:2110-21.

11 Hosseinpoor AR, Bergen N, Kunst A, et al. Socioeconomic inequalities in risk factors for non communicable diseases in low-income and middle-income countries: results from the World Health Survey. BMC Public Health 2012;12:912. 
12 International Institute for Population Sciences. Global adult tobacco survey GATS India 2009-2010. New Delhi: Ministry of Health and Family Welfare, Government of India, 2010.

13 McClernon FJ, Westman EC, Rose JE. The effects of controlled deep breathing on smoking withdrawal symptoms in dependent smokers. Addict Behav 2004:29:765-72.

14 Shahab L, Sarkar BK, West R. The acute effects of yogic breathing exercises on craving and withdrawal symptoms in abstaining smokers. Psychopharmacology (Berl) 2013;225:875-82.

15 Kochupillai V, Kumar P, Singh D, et al. Effect of rhythmic breathing (Sudarshan Kriya and Pranayam) on immune functions and tobacco addiction. Ann N Y Acad Sci 2005; 1056:242-52.

16 Craig P, Dieppe P, Macintyre S, et al. Developing and evaluating complex interventions: the new Medical Research Council guidance. BMJ 2008;337:a1655.

17 Sarkar BK, Shahab L, Arora M, et al. The social gradient in tobacco use does not generalise to low-income urban communities in India: findings from a census survey. Nicotine Tob Res 2016.

18 Arora M, Stigler M, Gupta V, et al. Tobacco control among disadvantaged youth living in low income communities in India: introducing project ACTIVITY. Asian Pacific J Cancer Prev 2010;11:7.

19 West R, Walia A, Hyder N, et al. Behavior change techniques used by the English Stop Smoking Services and their associations with short-term quit outcomes. Nicotine Tob Res 2010;12:742-7.

20 Sarkar BK, Shahab L, Arora M, et al. A cluster randomized controlled trial of a brief tobacco cessation intervention for low-income communities in India: study protocol. Addiction 2014;109:371-8.

21 Coleman T. ABC of smoking cessation. Use of simple advice and behavioural support. BMJ 2004;328:397-9.

22 West R, Hajek P, Stead L, et al. Outcome criteria in smoking cessation trials: proposal for a common standard. Addiction 2005;100:299-303.

23 Alterman Al, Gariti P, Niedbala RS. Varying results for immunoassay screening kits for cotinine level. Psychol Addict Behav 2002;16:256-9.

24 Heatherton TF, Kozlowski LT, Frecker RC, et al. Measuring the heaviness of smoking: using self-reported time to the first cigarette of the day and number of cigarettes smoked per day. Br J Addict 1989;84:791-9.
25 Russell MA, Wilson C, Taylor $C$, et al. Effect of general practitioners' advice against smoking. Br Med J 1979;2:231-5.

26 Brown J, Michie S, Geraghty AW, et al. Internet-based intervention for smoking cessation (StopAdvisor) in people with low and high socioeconomic status: a randomised controlled trial. Lancet Respir Med 2014;2:997-1006

27 Eysenbach G. The law of attrition. J Med Internet Res 2005;7:e11.

28 Murray DM, Varnell SP, Blitstein JL. Design and analysis of group-randomized trials: a review of recent methodological developments. Am J Public Health 2004:94:423-32.

29 Ma J, Raina P, Beyene J, et al. Comparison of population-averaged and cluster-specific models for the analysis of cluster randomized trials with missing binary outcomes: a simulation study. BMC Med Res Methodol 2013;13:9.

30 Donner $A$, Koval JJ. The estimation of intraclass correlation in the analysis of family data. Biometrics 1980;36:19-25.

31 Benjamini $Y$, Hochberg $Y$. Controlling the false discovery rate-a practical and powerful approach to multiple testing. J R Statist Soc, B 1995;57:289-300.

32 van Buuren $S$. Multiple imputation of discrete and continuous data by fully conditional specification. Stat Methods Med Res 2007;16:219-42.

33 Lancaster T, Stead LF. Self-help interventions for smoking cessation. Cochrane Database Syst Rev 2005(3):CD001118.

34 Stead LF, Hartmann-Boyce J, Perera R, et al. Telephone counselling for smoking cessation. Cochrane Database Syst Rev 2013;8:CD002850.

35 Bauld L, Judge K, Platt S. Assessing the impact of smoking cessation services on reducing health inequalities in England: observational study. Tob Control 2007;16:400-4.

36 Murthy $P$, Saddichha $S$. Tobacco cessation services in India: recent developments and the need for expansion. Indian J Cancer 2010;47(Suppl 1):69-74.

37 Stapleton JA, West R. A direct method and ICER tables for the estimation of the cost-effectiveness of smoking cessation interventions in general populations: application to a new cytisine trial and other examples. Nicotine Tob Res 2012;14:463-71.

38 Schrand JR. Does insular stroke disrupt the self-medication effects of nicotine? Med Hypotheses 2010;75:302-4.

39 Kumar MS, Sarma PS, Thankappan KR. Community-based group intervention for tobacco cessation in rural Tamil Nadu, India: a cluster randomized trial. J Subst Abuse Treat 2012;43:53-60. 